\title{
When Does an Additional Stage Improve Welfare in Centralized Assignment?
}

\author{
Battal Doğan \\ M. Bumin Yenmez
}

Discussion Paper 18 / 704

18 November 2018

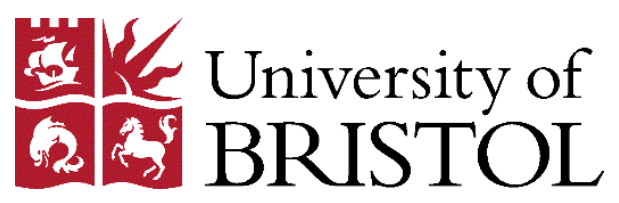

Department of Economics

University of Bristol

Priory Road Complex

Bristol BS8 1TU

United Kingdom 


\title{
WHEN DOES AN ADDITIONAL STAGE IMPROVE WELFARE IN CENTRALIZED ASSIGNMENT?
}

\author{
BATTAL DOĞAN AND M. BUMIN YENMEZ
}

\begin{abstract}
We study multistage centralized assignments to allocate scarce resources based on priorities in the context of school choice. We characterize the capacity-priority profiles of schools under which an additional stage of assignment may improve student welfare when the deferred acceptance algorithm is used at each stage. If the capacity-priority profile is acyclic, then no student prefers any subgame-perfect Nash equilibrium (SPNE) outcome of the 2-stage enrollment system to the truthful equilibrium outcome of the 1-stage enrollment system. If the capacity-priority profile is not acyclic, then an SPNE outcome of the 2-stage enrollment system may Pareto dominate the truthful equilibrium outcome of the 1-stage enrollment system.
\end{abstract}

\section{Introduction}

Centralized clearinghouses are used to allocate scarce resources when money cannot be used as a medium of exchange. For example, pupils are assigned to schools, families to public housing, and college students to courses and dormitory rooms. ${ }^{1}$ In this paper, we study when an additional stage of assignment improves welfare in centralized clearinghouses. We answer this question in the context of assigning students to public schools, the so-called school choice problem. In this context, families submit preferences that are used, together with school priorities, to create an assignment. In the last decade, many

Date: October 25, 2018.

Keywords: Market design, multistage assignment, school choice, deferred acceptance algorithm. Doğan is affiliated with the Department of Economics, University of Bristol, 3B13 The Priory Road Complex, Priory Road, Clifton, BS8 1TU, UK; Yenmez is with the Department of Economics, Boston College, 140 Commonwealth Ave, Chestnut Hill, MA, 02467, USA. Emails: battal.dogan@bristol.ac.uk, bumin. yenmez@bc. edu. We thank Lars Ehlers, Guillaume Haeringer, Bettina Klaus, Scott Duke Kominers, Alexey Kushnir, Vikram Manjunath, William Thomson, and participants at several seminars and conferences for comments. Battal Doğan gratefully acknowledges financial support from the Swiss National Science Foundation (SNSF) and the hospitality of the Center of Mathematical Sciences and Applications (CMSA, Harvard University) where part of this paper was written.

${ }^{1}$ There is a large market-design literature on assignment problems following the work of Shapley and Scarf (1974) and Hylland and Zeckhauser (1979). See Abdulkadiroğlu and Sönmez (2003) for school choice, Sönmez and Ünver (2010a) and Budish and Cantillon (2012) for course allocation, Sönmez and Switzer (2013) and Sönmez (2013) for cadet-branch matching, Schummer and Vohra (2013) for assignment of arrival slots, Echenique and Yenmez (2015) for controlled school choice, Delacrétaz et al. (2016) for refugee resettlement, and Combe et al. (2017) for teacher assignment. 
school districts have adapted the celebrated deferred acceptance (DA) algorithm of Gale and Shapley (1962) for this purpose.

The DA algorithm has a number of attractive properties. First of all, it is strategyproof in the sense that families do not have any incentives to misreport their preferences. Furthermore, it produces the student-optimal fair matching - every student finds the DA outcome at least as good as any other fair matching. However, these properties crucially depend on the assumption that there is no second stage of assignment to match students who are either unmatched in the first stage or who are not satisfied with their first-stage assignments. In practice, however, school districts have additional stages of assignment. For example, the newly adapted system in Chicago has two stages in which each student, at the end of the first stage, can accept his assigned school or reject it and participate in a second stage of assignment (Doğan and Yenmez, 2018).

To study the welfare effects of an additional stage, we assume that there are two stages of assignment, as in Chicago. At each stage, families submit preferences over schools and then DA is used to make an offer to students. Each student can accept the offer at the end of the first stage as their permanent assignment or reject it and participate at the second stage. We compare the equilibrium outcomes of this 2-stage enrollment system with the benchmark 1-stage enrollment system, which produces the DA outcome, and characterize when the introduction of a second stage may improve student welfare. A second stage can naturally improve welfare when there are frictions such as information asymmetries, application costs, limited choice for students, ${ }^{2}$ and so on. In contrast, we conduct our analysis in a model without any such frictions.

First, we consider the environment in which students truthfully submit their preferences at each stage in which they participate, but strategically accept or reject their offers. This environment paves the way for our subsequent analysis where students are more sophisticated and, in particular, they are also strategic in reporting their preferences. In addition, this environment provides several insights that are common with the environment in which students are more sophisticated.

We show that, when students are strategic only in acceptance-rejection decisions, any strategy-profile outcome, in particular any Nash equilibrium outcome, of the 2-stage enrollment system either Pareto dominates or is the same as the 1-stage enrollment outcome (Theorem 1). That is, an additional stage of assignment cannot hurt any student. This result is essentially driven by the following property of DA: When some students leave a problem with their DA assignments, no student is worse off in the DA outcome for the reduced problem (Lemma 1). This result also implies that it is a weakly dominant strategy to reject

\footnotetext{
${ }^{2}$ This is the so-called constrained school choice problem in which students can only rank up to a given number of schools, see Haeringer and Klijn (2009) and Calsamiglia et al. (2010).
} 
the first-stage offer at the 2-stage enrollment system. Hence, every student rejecting their first-stage offer is a Nash equilibrium of the 2-stage enrollment system, which produces the same outcome as the one-stage enrollment system.

When students are strategic only in acceptance-rejection decisions, although an additional stage of assignment cannot hurt any student, it is not guaranteed that an additional stage will improve welfare. We next identify conditions on capacity-priority profiles under which a second stage may improve welfare and conditions under which a second stage cannot improve welfare. We focus on conditions on the capacity-priority profile because school capacities and priorities are typically observable and commonly known, while students' preferences are their private information. The acyclicity condition on capacitypriority profiles, due to Ergin (2002), turns out to be critical. If the capacity-priority profile is acyclic, then any strategy profile of the 2-stage enrollment system is a Nash equilibrium that produces the same outcome as the 1-stage enrollment outcome; and, if the capacitypriority profile is not acyclic, then there exists a student preference profile such that a Nash equilibrium outcome of the 2-stage enrollment system Pareto dominates the 1-stage enrollment outcome (Theorem 2).

The critical nature of the acyclicity condition in how equilibrium outcomes of 1-stage and 2-stage enrollment systems compare to each other is essentially due to the fact that it is closely related to consistency: DA is consistent under a given capacity-priority profile if whenever some students leave a problem with their DA assignments, the assignments of the students at the DA outcome for the reduced problem does not change compared to their DA assignments at the original problem (Thomson, 1990). DA is consistent under a capacity-priority structure if, and only if, the capacity-priority structure is acyclic (Ergin, 2002). Given a problem, some students accepting their offers at the end of the first stage resembles a scenario where the same students leave the problem with their DA assignments and a new DA outcome is calculated for the remaining students and schools. In our setting, consistency is equivalent to acceptance and rejection strategies being outcome equivalent. Although consistency has been studied in the resource allocation literature mostly in reference to its normative appeal, in our setting consistency is important due to its relation to strategic behavior.

Second, we consider the environment in which students are also strategic in submitting their preferences. Since DA is strategy-proof, we assume that students submit their preferences truthfully and accept their offers at the second stage. Therefore, we consider the environment in which students strategically submit their preferences and decide whether to accept or reject their offers at the first stage. Unlike the first environment, a second stage may hurt a student at a subgame-perfect Nash equilibrium (SPNE). In fact, the 2-stage enrollment system may have an SPNE outcome at which all the students are 
worse off compared to the truthful equilibrium outcome of the 1-stage enrollment system (Example 3).

As before, we identify conditions on capacity-priority profiles under which a second stage may improve welfare and conditions under which a second stage cannot improve welfare. Again, the acyclicity condition on capacity-priority profiles is critical. If the capacity-priority profile is acyclic, then every student weakly prefers the truthful equilibrium outcome of the 1-stage enrollment system to any pure-strategy SPNE outcome of the 2-stage enrollment system (Theorem 3), and, if the capacity-priority profile is not acyclic, then there exists a preference profile such that an SPNE of the 2-stage enrollment system Pareto dominates the truthful equilibrium of the 1-stage enrollment system (Theorem 4). The intuition behind the acyclicity condition being critical for the equilibria comparisons is the same as the previous environment. The proofs to show that when acyclicity is violated, an SPNE of the 2-stage enrollment system Pareto dominates the truthful equilibrium of the 1-stage enrollment system in each environment, are both constructive, and the construction for the latter environment is more complex since profitable one-shot deviations must be avoided at more decisions.

Related Literature. In an influential study, Ergin (2002) shows that DA is consistent (or Pareto efficient) if, and only if, the capacity-priority profile is acyclic. Acyclic capacitypriority profiles play a key role in our analysis. We show that acyclicity of the capacitypriority profile is essential to how the DA outcome compares to the equilibrium outcomes of the 2-stage enrollment system. In a related study, Kesten (2010) introduces a modification of the DA algorithm that gives a Pareto efficient matching under any capacity-priority profile.

The distinguishing feature of our model is that the enrollment system may run in more than one stage. Manjunath and Turhan (2016) also consider a multistage enrollment system, where students submit preferences only once, at the beginning of the first stage. Furthermore, they assume that students participate at all stages. In contrast, a first-stage acceptance by a student is permanent in our 2-stage enrollment system. Furthermore, students are strategic in our setting. In a follow-up work, Turhan (2018) analyzes the mechanism introduced in Manjunath and Turhan (2016) for different partitions of schools from incentive and welfare perspectives.

Another paper that considers student assignment in multiple stages is Dur and Kesten (2018). Their main focus is understanding whether any sequential enrollment mechanism satisfying certain design goals such as non-wastefulness, strategy-proofness, fairness, and respecting improvements exists, while we take the mechanism to be used at each stage of the sequential process as given (DA) and compare the 2-stage and 1-stage enrollment system outcomes. In a recent work, Haeringer and Iehlé (2017) study college admissions in 
France, which features multiple stages of admissions. They introduce a dynamic stability notion and study mechanisms that satisfy this property.

Finally, Pycia (2012) studies non-cooperative games for the coalition formation problem and shows that if preferences are aligned, then there is a Nash equilibrium that produces the unique stable outcome (Proposition 5). Preference alignment imposes that if two agents are members of two different coalitions, then the agents should have the same preferences over the coalitions. In our setting, preference alignment cannot hold because a school and a student can be members of two different coalitions in which the student is indifferent between them (since there are no peer effects in our model) whereas the school can have strict preferences. Furthermore, our research question is the comparison of the two enrollment systems, which is different than that of Pycia.

\section{Model}

In this section, we formally introduce the primitives, notation, enrollment systems, and solution concepts.

2.1. Preliminary Definitions. Let $\mathcal{S}=\left\{s_{1}, \ldots, s_{|\mathcal{S}|}\right\}$ be a set of students and $\mathcal{C}=$ $\left\{c_{1}, \ldots, c_{|\mathcal{C}|}\right\}$ be a set of schools. Each student $s \in \mathcal{S}$ has a preference relation $R_{s}$ over $\mathcal{C} \cup\{s\},{ }^{3}$ where $s$ represents an outside option for the student, which can be a private school or homeschooling. Given $c, c^{\prime} \in \mathcal{C} \cup\{s\}$, we write $c P_{s} c^{\prime}$ if $c \neq c^{\prime}$ and $c R_{s} c^{\prime}$, i.e., student $s$ strictly prefers $c$ to $c^{\prime}$. A school $c$ is acceptable to student $s$ if the school is strictly more preferred than the outside option.

Each school $c \in \mathcal{C}$ has a capacity $q_{c} \in \mathbb{N}$, which represents the maximum number of students the school can admit and a priority ranking $\succeq_{c}$ over the set of students $\mathcal{S} \cup\{c\}$. Here, $c$ denotes the option of having an empty seat. The strict part of the priority ranking $\succeq_{c}$ is denoted as $\succ_{c}$, so, for any $s, s^{\prime} \in \mathcal{S} \cup\{c\}$, if $s \succeq_{c} s^{\prime}$ and $s \neq s^{\prime}$, then $s \succ_{c} s^{\prime}$. A student $s$ is acceptable to school $c$ if $s \succ_{c} c$.

The admissions policy of each school $c \in \mathcal{C}$ is represented by a choice function $C h_{c}$ : $2^{\mathcal{S}} \times\left\{1, \ldots, q_{c}\right\} \rightarrow 2^{\mathcal{S}}$, which maps each nonempty set $S \subseteq \mathcal{S}$ of students and each $q \in\left\{1, \ldots, q_{c}\right\}$ to a subset $C h_{c}(S ; q) \subseteq S$ of chosen students such that $\left|C h_{c}(S ; q)\right| \leq q$. Here, $q$ represents the remaining capacity, so the school cannot admit more than $q$ students. In a 2-stage enrollment system, the admissions policy may depend on the number of seats available since a school needs to specify how to allocate the remaining seats at the second stage after some of the seats are allocated in the first stage. We assume that for each

\footnotetext{
${ }^{3}$ More formally, a preference relation over $\mathcal{C} \cup\{s\}$ is a complete, transitive, and anti-symmetric binary relation over $\mathcal{C} \cup\{s\}$. Binary relation $R_{s}$ over $\mathcal{C} \cup\{s\}$ is complete if, for every $c_{1}, c_{2} \in \mathcal{C} \cup\{s\}, c_{1} R_{s} c_{2}$ or $c_{2} R_{s} c_{1}$. It is transitive if, for every $c_{1}, c_{2}, c_{3} \in \mathcal{C} \cup\{s\}, c_{1} R_{s} c_{2}$ and $c_{2} R_{s} c_{3}$ imply $c_{1} R_{s} c_{3}$. It is anti-symmetric if, for every $c_{1}, c_{2} \in \mathcal{C} \cup\{s\}, c_{1} R_{s} c_{2}$ and $c_{2} R_{s} c_{1}$ imply $c_{1}=c_{2}$.

${ }^{4}$ The priority ranking $\succeq_{c}$ is a complete, transitive, and anti-symmetric binary relation over $\mathcal{S} \cup\{c\}$.
} 
school $c \in \mathcal{C}, C h_{c}$ is responsive to the priority ranking $\succeq_{c}$, i.e., for each $S \subseteq \mathcal{S}, C h_{c}(S ; q)$ is obtained by choosing the highest-priority acceptable students in $S$ until $q$ students are chosen or no acceptable student is left.

A (school choice) problem is a tuple $\left(\mathcal{S}, \mathcal{C},\left(R_{s}\right)_{s \in \mathcal{S}},\left(q_{c}, \succeq_{c}\right)_{c \in \mathcal{C}}\right)$. Sometimes, we fix all the primitives of a problem other than the student preference-relation profile; in that case, we refer to the student-preference relation profile $\left(R_{s}\right)_{s \in \mathcal{S}}$ as the problem.

A matching $\mu$ is an assignment of students to schools respecting the capacity constraints:

- for each student $s, \mu(s) \in \mathcal{C}$ or $\mu(s)=s$,

- for each school $c, \mu(c) \subseteq \mathcal{S}$ and $|\mu(c)| \leq q_{c}$, and

- for each student $s$ and school $c, \mu(s)=c$ if, and only if, $s \in \mu(c)$.

In words, every student is either matched with a school or matched to his outside option (which we also refer to as being unmatched), and every school is assigned a set of students with cardinality less than or equal to its capacity. In addition, there is a feasibility constraint so that if a student gets matched with a school, then the student is in the set of students that is assigned to the school.

A matching $\mu$ Pareto dominates another matching $v$, if, for every student $s, \mu(s)$ is weakly more preferred than $v(s)$, and, for one student, it is strictly more preferred. A matching that is not Pareto dominated is called Pareto efficient.

A matching $\mu$ is stable if it satisfies the following properties:

- (individual rationality for students) for each student $s, \mu(s) R_{s} s$,

- (individual rationality for schools) for each school $c, C h_{c}\left(\mu(c) ; q_{c}\right)=\mu(c)$, and

- (no blocking) there exists no student-school pair $(s, c)$ such that $c P_{s} \mu(s)$ and $s \in C h_{c}\left(\mu(c) \cup\{s\} ; q_{c}\right)$.

Individual rationality for a student means that she weakly prefers the outcome to the outside option. On the other hand, for a school, it means that the school would like to keep all the students assigned to it. More explicitly, since choice functions are responsive, only acceptable students are matched with the school and the number of students is no more than its capacity. No blocking rules out the existence of a student-school pair such that the student strictly prefers the school to his match and the school would like to admit the student. In student-assignment settings, the stability of a matching is viewed as a fairness notion (Abdulkadiroğlu and Sönmez, 2003).

A stable matching is student optimal if every student weakly prefers the outcome in this matching to the outcome in any other stable matching.

An assignment rule associates each problem with a matching. The deferred acceptance (DA) rule due to Gale and Shapley (1962) is used in many school districts that have reformed their school choice systems. The DA rule associates each problem with the matching determined by the following deferred acceptance algorithm. 
Step 1. Each student applies to his top-ranked acceptable school. If there is no such school, then she is unmatched. Each school $c$ considers its applicants, say $A_{1}(c)$. Among these, it tentatively accepts $C h_{c}\left(A_{1}(c) ; q_{c}\right)$. It rejects all the other applicants. If there is no rejection by any school at this step, then stop and return $\cup_{c \in \mathcal{C}} C h_{c}\left(A_{1}(c) ; q_{c}\right)$.

Step $t \geq 2$. Each student who is rejected at Step $t-1$ applies to his topranked acceptable school among the ones that have not rejected him. If there is no such school, she is unmatched. Each school $c$ considers the students that it tentatively accepted at Step $t-1$ and the new applicants at Step $t$, say $A_{t}(c)$. Among these, it tentatively accepts $C h_{c}\left(A_{t}(c) ; q_{c}\right)$. It rejects all the other applicants. If there is no rejection by any school at this step, then stop and return $\cup_{c \in \mathcal{C}} C h_{c}\left(A_{t}(c) ; q_{c}\right)$.

The algorithm stops in finite time since there can only be a finite number of rejections. The DA algorithm produces the student-optimal stable matching (Gale and Shapley, 1962).

2.2. Enrollment Systems and Games. The student enrollment systems in many districts have supplementary stages for students who are unmatched or matched with lesspreferred schools. Thus, we consider two centralized enrollment systems depending on whether the enrollment system has two stages or only one stage.

1-Stage Enrollment System: Each student reports a preference relation. Then, a matching is determined by the DA rule. Finally, each student either accepts her match or rejects it (in which case she is matched to her outside option).

2-Stage Enrollment System: In the first stage, a matching is determined through the 1stage enrollment system described above. In the second stage, if there are any remaining students (those who were unmatched or rejected their matches at the end of the first stage) and there are available seats, another matching is determined through the 1-stage enrollment system among the unmatched students and schools with reduced capacities.

We assume that students may be strategic when reporting their preferences or making their acceptance/rejection decisions, but schools are not strategic, and their priority rankings and capacities are common knowledge. Each enrollment system, at each preference profile, induces an extensive-form game. We study the Nash equilibria and subgame-perfect Nash equilibria (SPNE) of these games and compare the equilibria of the two enrollment systems.

In the extensive-form game at a given preference profile, a student's strategy should recommend, at each stage, what preference relation to report and whether to accept a matched school as a function of the history. Let us call a student's strategy truthful if it 
recommends reporting, at each stage and for each history, the true preference relation. Note that there can be many different truthful strategies, which differ in the acceptance-rejection recommendations but always report the preferences truthfully.

We say that a student's strategy weakly dominates another strategy if, for each strategy profile of the other students, the former strategy yields an outcome that is weakly preferred by the student to the outcome of the latter strategy. ${ }^{5}$ We say that two student strategies are outcome equivalent if, for each strategy profile of the other students, the student is indifferent between the outcomes of the two strategies.

It is a weakly dominant strategy for each student to truthfully report his preferences in DA (Dubins and Freedman, 1981). In other words, the game induced by DA has a truthful Nash equilibrium with weakly-dominant strategies. As a result, most of the literature focuses on this particular equilibrium, even though the game induced by DA may have other Nash equilibria. ${ }^{6}$

Under the 1-stage enrollment system, it is a weakly dominant strategy to accept any offer that is weakly more preferred than the outside option. Therefore, there is a truhful SPNE in which students accept their offers. We also focus on this truthful equilibrium of the 1-stage enrollment system.

2.3. Consistency and Acyclic Capacity-Priority Profiles. An assignment rule is consistent under a given capacity-priority profile if, for each problem with that capacity-priority profile and for each reduced problem obtained by the departure of an arbitrary subgroup of students with their assigned seats at the original problem, the matching outcome of the assignment rule for the reduced problem is the restriction of the matching chosen for the original problem to that subgroup.

More formally, given a problem $\left(\mathcal{S}, \mathcal{C},\left(R_{S}\right)_{s \in \mathcal{S}},\left(q_{c}, \succeq_{c}\right)_{c \in \mathcal{C}}\right)$, a set of students $S \subseteq \mathcal{S}$, and a matching $\mu$, let $\left.\left(\mathcal{S}, \mathcal{C},\left(R_{S}\right)_{s \in \mathcal{S}},\left(q_{c}, \succeq_{c}\right)_{c \in \mathcal{C}}\right)\right|_{(S, \mu)}$ denote the reduced problem when students in $S$ leave the problem with their assigned seats in $\mu$. We say that an assignment rule $\varphi$ is consistent under the capacity-priority profile $\left(q_{c}, \succeq_{c}\right)_{c \in \mathcal{C}}$ if, for each preference profile $R$, set of students $S \subseteq \mathcal{S}$, and student $s \in \mathcal{S} \backslash S$, we have $\varphi_{s}\left(\mathcal{S}, \mathcal{C},\left(R_{S}\right)_{s \in \mathcal{S}},\left(q_{c}, \succeq_{c}\right.\right.$

\footnotetext{
${ }^{5}$ This is the definition of weak domination used in mechanism design (Jackson, 2003) and matching theory (Roth and Sotomayor, 1990, Definition 4.2). However, in the game-theory literature, weak domination also requires that there exists a strategy profile of the other students for which the outcome of the former strategy is strictly preferred to the outcome of the latter strategy.

${ }^{6}$ This equilibrium has been commonly used in the literature in the analysis of DA. For instance, Ergin and Sönmez (2006) analyze Nash equilibrium outcomes of the game induced by another well-known system, the Boston mechanism, and compare those outcomes with the truthful equilibrium outcome of DA. Yet, DA may have other Nash equilibria, even with an unstable outcome (Sotomayor, 2008). Furthermore, Bando (2014) shows that the outcome of the efficiency-adjusted deferred acceptance mechanism (Kesten, 2010) is a strict Nash equilibrium of DA, which is weakly more preferred than the truthful equilibrium outcome of DA for every student.
} 
)$\left._{c \in \mathcal{C}}\right)=\varphi_{s}\left(\left.\left(\mathcal{S}, \mathcal{C},\left(R_{s}\right)_{s \in \mathcal{S}},\left(q_{c}, \succeq_{c}\right)_{c \in \mathcal{C}}\right)\right|_{\left(S, \varphi\left(\mathcal{S}, \mathcal{C},\left(R_{s}\right)_{s \in \mathcal{S}},\left(q_{c}, \succeq_{c}\right)_{c \in \mathcal{C}}\right)\right)}\right)$. We say that an assignment rule is consistent if it is consistent under any capacity-priority profile (Thomson, 1990).

A capacity-priority profile $\left(q_{c}, \succeq_{c}\right)_{c \in \mathcal{C}}$ includes a cycle if there exist a pair of schools $c$ and $c^{\prime}$, and three students $s, s^{\prime}$, and $s^{\prime \prime}$ such that

i. $s \succ_{c} s^{\prime} \succ_{c} s^{\prime \prime} \succ_{c^{\prime}} s$ and

ii. there exist (possibly empty) disjoint sets of students $S_{c}, S_{c^{\prime}} \subset \mathcal{S} \backslash\left\{s, s^{\prime}, s^{\prime \prime}\right\}$ such that $S_{c} \subset\left\{i \in S: i \succ_{c} s^{\prime}\right\}, S_{c^{\prime}} \subset\left\{i \in S: i \succ_{c^{\prime}} s\right\},\left|S_{c}\right|=q_{c}-1$, and $\left|S_{c^{\prime}}\right|=q_{c^{\prime}}-1$.

A capacity-priority profile $\left(q_{c}, \succeq_{c}\right)_{c \in \mathcal{C}}$ is acyclic if it does not include any cycle. Ergin (2002) shows that DA is consistent (or Pareto efficient) under the capacity-priority profile $\left(q_{c}, \succeq_{c}\right)_{c \in \mathcal{C}}$ if, and only if, $\left(q_{c}, \succeq_{c}\right)_{c \in \mathcal{C}}$ is acyclic. Acyclic capacity-priority profiles play a crucial role in our analysis.

The following interpretation of acyclicity provides some intuition. Given a problem $\left(\mathcal{S}, \mathcal{C},\left(R_{s}\right)_{s \in \mathcal{S}},\left(q_{c}, \succeq_{c}\right)_{c \in \mathcal{C}}\right)$, we say that student $s$ is an interrupter at school $c$ if there is a step of the DA algorithm where student $s$ is tentatively accepted by school $c$ but another student is rejected, while at a later step student $s$ is also rejected. Note that according to this definition, student $s$ could be accepted by the school for the first time at an earlier step without rejecting any student, but the school may reject another student at a later step while still accepting student $s$. In this case, student $s$ would still be an interrupter if she is rejected at a still later step. Kesten (2010) shows that a problem with the capacity-priority profile $\left(q_{c}, \succeq_{c}\right)_{c \in \mathcal{C}}$ does not have an interrupter if, and only if, $\left(q_{c}, \succeq_{c}\right)_{c \in \mathcal{C}}$ is acyclic.

\section{Students Are Strategic Only in Acceptance-rejection Decisions}

To compare the outcomes of the 1-stage and 2-stage enrollment systems, we first consider the case when students use truthful strategies: Suppose that each student reports her preferences truthfully in the first stage, and also in the second stage, if she rejects her offer at the end of the first stage. In addition, accepting the offer after the second stage is optimal for students, so the only strategic decision for a student is the acceptance-rejection decision at the end of the first stage.

Consider the game in which students observe their assigned schools at the first stage and decide whether to accept or reject an offer, and students who reject their offers move to the second stage, where they report truthfully and accept their offers. At each problem, this game can be represented by a one-stage simultaneous-move game where the strategy set of each student consists of accepting the first-stage offer or rejecting it.

Theorem 1. Suppose that students are strategic only in acceptance-rejection decisions. Then any strategy profile outcome, in particular any Nash equilibrium outcome, of the 2-stage enrollment system either Pareto dominates or is the same as the 1-stage enrollment outcome. 
To show this result, we use the following property of DA. Given a problem, consider the DA outcome. Let a set of students leave the problem with their assigned seats in DA, reducing the capacities of the matched schools. Run DA again in the reduced problem. In this setting, it is known that the matching for the remaining students may change, i.e., DA may not be consistent, unless the capacity-priority profile is acyclic (Ergin, 2002). Lemma 1 shows that each student remaining in the problem gets a weakly more preferred school (Chen, 2017). This implies that rejecting the offer after the first stage is a weakly dominant strategy since at the second stage every student is matched with a weakly more preferred school, regardless of what other students decide. Hence, at any strategy profile of the 2-stage system, a student either accepts his offer at the first stage and is assigned to his 1-stage enrollment school, or some students leave with their 1-stage enrollment schools and in the second stage the student is assigned a school that he weakly prefers to his 1-stage enrollment school.

Also note that in the 2-stage enrollment system, all students rejecting their first-stage offers is a Nash equilibrium, which produces the same outcome as the 1-stage enrollment system.

The following example illustrates that a Nash equilibrium outcome of the 2-stage enrollment system may indeed Pareto dominate the 1-stage enrollment outcome when the capacity-priority profile has a cycle (i.e., it is not acyclic).

Example 1. Let $\mathcal{S}=\left\{s_{1}, s_{2}, s_{3}\right\}$ and $\mathcal{C}=\left\{c_{1}, c_{2}, c_{3}\right\}$. Each school has capacity one. Let the student preference profile $\left(R_{s}\right)_{s \in \mathcal{S}}$ and the school priority profile $\left(\succeq_{c}\right)_{c \in \mathcal{C}}$ be as below.

\begin{tabular}{ccccccc}
$R_{s_{1}}$ & $R_{s_{2}}$ & $R_{s_{3}}$ & & $\succeq_{c_{1}}$ & $\succeq_{c_{2}}$ & $\succeq_{c_{3}}$ \\
\cline { 1 - 3 }$c_{1}$ & $c_{1}$ & $c_{2}$ & & $s_{3}$ & $s_{2}$ & $s_{1}$ \\
$c_{3}$ & $c_{2}$ & $c_{1}$ & & $s_{1}$ & $s_{3}$ & $s_{2}$ \\
$c_{2}$ & $c_{3}$ & $c_{3}$ & & $s_{2}$ & $s_{1}$ & $s_{3}$ \\
$s_{1}$ & $s_{2}$ & $s_{3}$ & & $c_{1}$ & $c_{2}$ & $c_{3}$
\end{tabular}

In the first stage, student $s_{1}$ gets an offer from school $c_{3}$, student $s_{2}$ gets an offer from school $c_{2}$, and student $s_{3}$ gets an offer from school $c_{1}$. Consider the strategy profile where student $s_{1}$ accepts her offer and the other two students reject their offers. The outcome of this strategy profile is that student $s_{1}$ gets school $c_{3}$, student $s_{2}$ gets school $c_{1}$, and student $s_{3}$ gets school $c_{2}$. Since students $s_{2}$ and $s_{3}$ get their best schools, they do not have any profitable unilateral deviation. If student $s_{1}$ instead rejects, he still receives school $c_{3}$, so he also does not have a profitable unilateral deviation. Therefore, we have a Nash equilibrium outcome that Pareto dominates the 1-stage enrollment outcome in which student $s_{1}$ gets school $c_{3}$, student $s_{2}$ gets school $c_{2}$, and student $s_{3}$ gets school $c_{1}$. 
We next show that a capacity-priority profile with a cycle is necessary and sufficient for the 2-stage enrollment system to feature a Nash equilibrium outcome which Pareto improves upon the 1-stage enrollment outcome.

Theorem 2. Suppose that students are strategic only in acceptance-rejection decisions.

i. If the capacity-priority profile is acyclic, then any strategy profile of the 2-stage enrollment system is a Nash equilibrim, which produces the same outcome as the 1-stage enrollment outcome.

ii. If the capacity-priority profile has a cycle, then there exists a student preference profile such that a Nash equilibrium outcome of the 2-stage enrollment system Pareto dominates the 1-stage enrollment outcome.

The proof of Theorem 2 is provided in the Appendix. Part $i$ follows from the fact that DA is consistent under an acyclic capacity-priority profile and therefore, for any student, accepting the first stage offer or rejecting it are outcome equivalent. The proof of Part $i i$ is constructive. The intuition can be given using Example 1, which has a cycle in the capacity-priority profile because $s_{3} \succ_{c_{1}} s_{1} \succ_{c_{1}} s_{2} \succ_{c_{2}} s_{3}$ and school capacities are one. Student $s_{1}$ is an interrupter because student $s_{2}$ is rejected while student $s_{1}$ is tentatively accepted, and student $s_{1}$ is eventually rejected as well. If we consider the equilibrium strategy profile in which the interrupter student $s_{1}$ accepts her offer and the other two students reject their offers, both students $s_{2}$ and $s_{3}$ get strictly more preferred schools.

Remark 1. The first statement in Theorem 2 holds for any assignment rule that is consistent under a capacity-priority profile. Kesten (2006) characterizes capacity-priority profiles under which the top trading cycles rule is consistent. Kojima and Ünver (2014) and Doğan and Klaus (2018) characterize the Boston (or immediate acceptance) rule with some desirable properties which include consistency. Ergin (2000) studies consistent sequential rules for house allocation problems. ${ }^{7}$ Therefore, our result holds for the top trading cycles rule under the assumptions stated in Kesten (2006), the sequential rules, and the Boston rule.

Remark 2. When students are strategic only in acceptance-rejection decisions, Theorems 1 and 2 hold for the comparison between $k$-stage and 1-stage enrollment systems for every $k \geq 2$. Furthermore, if $k$ is sufficiently large, there exists a pure-strategy SPNE of the $k$-stage enrollment system that has the outcome of the efficiency-adjusted deferred acceptance algorithm (Kesten, 2010), which is Pareto efficient.

\footnotetext{
${ }^{7}$ See also Sönmez and Ünver (2010b) for house allocation problem with existing tenants.
} 


\section{Students Are Strategic Also When Reporting First-stage Preferences}

Suppose now that students are strategic also when reporting their first-stage preferences, in addition to the acceptance-rejection decisions at the end of the first stage.

Consider the complete information game where students report preference relations, then observe the reported preferences at the first stage (and also the profile of offers), and decide whether to accept an offer or not, and the students who reject their offers move to the second stage, where they report truthfully and accept the new offer. At each problem $\left(R_{S}\right)_{S \in \mathcal{S}}$, this game can be represented by an extensive-form complete-information game where a student strategy is a complete-contingent plan that specifies which preference relation to report and whether to accept or reject the offer following each possible reported preference profile. We compare the SPNE of this game with the truthful equilibrium of the 1-stage enrollment system.

The following example illustrates that an SPNE outcome of the 2-stage enrollment system may Pareto dominate the 1-stage enrollment system outcome.

Example 2. Consider the school choice problem in Example 1 again. The 1-stage enrollment outcome is that student $s_{1}$ gets school $c_{3}$, student $s_{2}$ gets school $c_{2}$, and student $s_{3}$ gets school $c_{1}$. Consider the following strategy profile. Student $s_{1}$ reports only school $c_{3}$ as acceptable in the first stage, and accepts an offer if, and only if, it is strictly more preferred than her 1-stage enrollment outcome school $c_{3}$. Each other student reports truthfully in the first stage and at each possible preference profile realization, and they accept an offer if, and only if, it is strictly more preferred than their 1-stage enrollment outcome.

Consider student $s_{1}$. Consider her preference-revelation decision node. By changing her preference report, she either causes an outcome where student $s_{2}$ gets school $c_{2}$ and student $s_{3}$ gets school $c_{1}$, or an outcome where student $s_{2}$ gets school $c_{1}$ and student $s_{3}$ gets school $c_{2}$. In the former case, all students reject their offers, and student $s_{1}$ in the end receives school $c_{3}$. In the latter case, only student $s_{1}$ rejects her offer, and in the end she still receives school $c_{3}$. So there is no one-shot profitable deviation at her preference-revelation decision node. Consider any of her acceptance-rejection decision nodes. If she is offered school $c_{1}$, she clearly cannot be strictly better off by rejecting it. If she is offered school $c_{3}$ and she rejects it, in the worse case she receives school $c_{3}$ in the second stage, so again there is no one-shot profitable deviation. If she receives school $c_{2}$ and rejects it, then she receives a school at the second stage that cannot be strictly less preferred than school $c_{2}$. Again, there is no profitable one-shot deviation.

Consider student $s_{2}$. Consider his preference-revelation decision node. Clearly, there is no one-shot profitable deviation since he gets his best school. Consider any of his acceptance-rejection decision nodes. If he is offered school $c_{1}$, he can clearly not be better off by rejecting the offer. If he is offered school $c_{2}$ and he rejects it, in the worse case he 
receives school $c_{2}$ in the second stage, so there is no one-shot profitable deviation either. If he is offered school $c_{3}$ in the first stage, by rejecting this offer he gets a school at the second stage that cannot be worse than school $c_{3}$. Again there is no profitable one-shot deviation.

The case for student $s_{3}$ is symmetrical to the case for student $s_{2}$. Thus, student $s_{3}$ does not have a profitable one-shot deviation either.

Therefore, an SPNE outcome of the 2-stage enrollment system may Pareto dominate the truthful equilibrium outcome of the 1-stage enrollment system. We show that this is not possible under an acyclic capacity-priority profile. In fact, we prove a stronger result:

Theorem 3. Suppose that the capacity-priority profile is acyclic. Then every student weakly prefers the truthful equilibrium outcome of the 1-stage enrollment system to any pure-strategy SPNE outcome of the 2-stage enrollment system.

In particular, when the capacity-priority profile is acyclic, an SPNE outcome of the 2-stage enrollment system is either the same as the truthful equilibrium outcome of the 1-stage enrollment system, or it is Pareto dominated by the latter. To show this result, we first show that an SPNE outcome of the 2-stage enrollment system cannot have a blocking pair. The intuition is that, if the priority of student $s$ is violated by another student $s^{\prime}$ at a school $c$, then students $s$ and $s^{\prime}$ must be receiving their final assignments at different stages of the 2-stage enrollment system. Moreover, student $s$, by a one-shot deviation, can instead participate at the stage where student $s^{\prime}$ receives school $c$, and can guarantee himself at least school $c$, since a lower-priority student is receiving $c$ before his arrival into the problem. Here, the acyclic capacity-priority profile assumption is crucial because, otherwise, student $s$ could have been an interrupter in the new problem and could have ended up with a strictly less-preferred school than $c$, although without his presence a lower-priority student is able to receive school $c$. Once we show that there is no blocking pair, the rest follows from the fact that the 1-stage enrollment outcome is the student-optimal stable matching.

The next result shows that the acyclic capacity-priority profile assumption is necessary for the previous result that students weakly prefer the 1-stage enrollment system outcome to any pure-strategy SPNE outcome of the 2-stage enrollment system.

Theorem 4. Suppose that the capacity-priority profile has a cycle. Then there exists a preference profile such that an SPNE of the 2-stage enrollment system Pareto dominates the truthful equilibrium of the 1-stage enrollment system.

The proof of Theorem 4, which is provided in the Appendix, is constructive. The construction builds upon the idea in Example 2 and resembles the construction in the proof of Theorem 2. Yet, the constructed strategy profile is more complex than the constructed 
strategy profile in the proof of Theorem 2 since profitable one-shot deviations at many more decisions nodes must be avoided.

Unlike the case when the students are strategic only in acceptance-rejection decisions, the truthful equilibrium outcome of the 1-stage enrollment system may indeed Pareto dominate a pure-strategy SPNE outcome of the 2-stage enrollment system, as illustrated by the following example.

Example 3. Let $\mathcal{S}=\left\{s_{1}, s_{2}, s_{3}\right\}$ and $\mathcal{C}=\left\{c_{1}, c_{2}, c_{3}\right\}$. Each school has capacity one. The student preference profile and school priority profile can be depicted as below.

\begin{tabular}{|c|c|c|c|c|c|}
\hline & $R_{S_{2}}$ & $R_{S_{3}}$ & $\tau_{c}$ & $\succeq_{c}$ & $\succ$ \\
\hline$c_{1}$ & $c_{2}$ & $c_{3}$ & $s_{3}$ & $s_{1}$ & \\
\hline$c_{2}$ & $c_{3}$ & $c_{1}$ & $s_{2}$ & $s_{3}$ & \\
\hline$s_{1}$ & $s_{2}$ & $s_{3}$ & $s_{1}$ & $s_{2}$ & \\
\hline$c_{3}$ & $c_{1}$ & $c_{2}$ & $c_{1}$ & $c_{2}$ & \\
\hline
\end{tabular}

In the 1-stage enrollment system, each student is matched with his top-ranked school: student $s_{1}$ is matched with school $c_{1}$, student $s_{2}$ is matched with school $c_{2}$, and student $s_{3}$ is matched with school $c_{3}$.

Let $R^{\prime}$ be a preference profile such that student $s_{1}$ finds only school $c_{2}$ acceptable at $R_{s_{1}}^{\prime}$, student $s_{2}$ finds only school $c_{3}$ acceptable at $R_{s_{2}}^{\prime}$, and student $s_{3}$ finds only school $c_{1}$ acceptable at $R_{s_{3}}^{\prime}$. Consider the following strategy profile for the 2-stage enrollment system: Each student $s$ reports $R_{s}^{\prime}$ in the first stage and accepts his offer following a preference profile realization $\bar{R}$ if, and only if, $\bar{R}_{s}=R_{s}^{\prime}$ and there is at least one other student $s^{\prime} \in \mathcal{S} \backslash\{s\}$ such that $\bar{R}_{s^{\prime}}=R_{s^{\prime}}^{\prime}$.

Note that the outcome of this strategy profile is that student $s_{1}$ is matched with school $c_{2}$, student $s_{2}$ is matched with school $c_{3}$, and student $s_{3}$ is matched with school $c_{1}$, which is Pareto dominated by the 1-stage enrollment outcome. We show that this strategy profile is an SPNE. Consider student $s_{1}$. Since student $s_{2}$ has the top priority at school $c_{3}$ and student $s_{3}$ has the top priority at school $c_{1}$, by a one-shot deviation at his preference-revelation decision node student $s_{1}$ cannot affect the assignments of students $s_{2}$ or $s_{3}$, but can only change his own assignment from school $c_{2}$ to his outside option $s_{1}$. At the given strategy profile, students $s_{2}$ and $s_{3}$ accept their offers at the first stage, student $s_{1}$ rejects his offer and in the second stage he receives school $c_{2}$. Thus, student $s_{1}$ does not have a profitable one-shot deviation at his preference-revelation decision node.

Consider the acceptance-rejection decision node of student $s_{1}$ following an arbitrary preference profile realization $\bar{R}$. Suppose that $\bar{R}$ is such that at the given strategy profile, it recommends student $s_{1}$ to reject his offer at the acceptance-rejection decision node following $\bar{R}$. There are two possible cases. The first case is that $\bar{R}_{s_{1}} \neq R_{s_{1}}^{\prime}, \bar{R}_{s_{2}}=R_{s_{2}}^{\prime}$, and $\bar{R}_{s_{3}}=R_{s_{3}}^{\prime}$. In this case, the first-stage offer of student $s_{1}$ is either school $c_{2}$ or his outside 
option $s_{1}$, and the strategy recommends student $s_{1}$ to reject his offer, student $s_{2}$ to accept school $c_{3}$, and student $s_{3}$ to accept school $c_{1}$. Clearly, student $s_{1}$ does not have a profitable one-shot deviation at this decision node. The second case is that all the students reject their offers at their decision nodes following $\bar{R}$. In this case, student $s_{1}$ is assigned to his top-ranked school $c_{1}$ and there is no profitable one-shot deviation.

Suppose that $\bar{R}$ is such that at the given strategy profile, it recommends student $s_{1}$ to accept his offer at the acceptance-rejection decision node following $\bar{R}$. There are three possible cases. The first case is that for each student $s \in \mathcal{S}, \bar{R}_{s}=R_{s}^{\prime}$, in which case student $s_{1}$ clearly does not have a profitable one-shot deviation at this decision node. The second case is that $\bar{R}_{s_{1}}=R_{s_{1}}^{\prime}, \bar{R}_{s_{2}}=R_{s_{2}}^{\prime}$, and $\bar{R}_{s_{3}} \neq R_{s_{3}}^{\prime}$. In this case, if student $s_{1}$ rejects his offer by a one-shot deviation at this decision node, in the second stage he will still be assigned to school $c_{2}$ since his more preferred school $c_{1}$ will be assigned to student $s_{3}$, who has a higher priority at school $c_{1}$, which is therefore not a profitable deviation. The third case is that $\bar{R}_{s_{1}}=R_{s_{1}}^{\prime}, \bar{R}_{s_{2}} \neq R_{s_{2}}^{\prime}$, and $\bar{R}_{s_{3}}=R_{s_{3}}^{\prime}$. In this case, if student $s_{1}$ rejects his offer by a one-shot deviation at this decision node, he will still be assigned to school $c_{2}$ in the second stage since his more preferred school $c_{1}$ will be taken by student $s_{3}$ in the first stage, which is therefore not a profitable deviation.

Hence, student $s_{1}$ has no profitable one-shot deviation. By symmetrical arguments, no student has a profitable one-shot deviation.

Even though the 2-stage enrollment system has a pure-strategy Nash equilibrium when students are only strategic in acceptance-rejection decisions, it does not necessarily have a pure-strategy SPNE when students are also strategic in submitting their first-stage preferences. Note that each subgame that follows a preference-revelation profile resembles an allocation problem where each student has an endowment school (the school that she is assigned to at the first stage) and has to decide on whether to stay out of the second stage and enjoy her endowment or stay in together with her endowment. In the second stage, a student can possibly be assigned a strictly more preferred school than her endowment, which is an incentive to reject the first-stage offer. However, a school being your endowment does not provide any additional priority at that school in the second stage, which is an incentive to accept the first-stage offer. The following example shows that there exists a subgame where these incentives cannot be balanced, or in particular there exists a problem at which the 2-stage enrollment system does not have a pure-strategy SPNE even when all schools have the same priority ranking of students.

Example 4. Let $\mathcal{S}=\left\{s_{1}, s_{2}, s_{3}, s_{4}\right\}$ and $\mathcal{C}=\left\{a, \omega\left(s_{1}\right), \omega\left(s_{2}\right), \omega\left(s_{3}\right), \omega\left(s_{4}\right)\right\}$. Each school has capacity one. Let the student preference profile $R$ and the school priority profile $\succeq$ be depicted as below. The part of the preference profile denoting the unacceptable schools are not relevant and not depicted. School have a common priority ordering over students. 


\begin{tabular}{cccccc}
$R_{s_{1}}$ & $R_{s_{2}}$ & $R_{s_{3}}$ & $R_{s_{4}}$ & & $\left(\succeq_{c}\right)_{c \in \mathcal{C}}$ \\
\cline { 1 - 4 }$\left(s_{3}\right)$ & $\omega\left(s_{4}\right)$ & $a$ & $a$ & & $s_{1}$ \\
$\omega\left(s_{1}\right)$ & $a$ & $\omega\left(s_{3}\right)$ & $\omega\left(s_{4}\right)$ & & $s_{2}$ \\
$s_{1}$ & $\omega\left(s_{2}\right)$ & $s_{3}$ & $s_{4}$ & & $s_{3}$ \\
& $s_{2}$ & & & & $s_{4}$
\end{tabular}

Consider the subgame that follows after student $s_{i}$ reports school $\omega\left(s_{i}\right)$ as the only acceptable school, for each $i \in\{1, \ldots, 5\}$. Note that student $s_{i}$ gets an offer from school $\omega\left(s_{i}\right)$ at the first stage, for each $i \in\{1, \ldots, 5\}$. Suppose that that this subgame has a pure strategy Nash equilibrium, say $\sigma$.

Since $a P_{s_{2}} \omega\left(s_{2}\right)$ and student $s_{2}$ has the top priority from among the students who find $a$ acceptable, it must be that student $s_{2}$ rejects his offer at $\sigma$ since he can guarantee himself at least $a$ by participating in the second stage. Now, we claim that student $s_{4}$ must accept his offer, $\omega\left(s_{4}\right)$, at $\sigma$. Suppose not, that is, suppose that student $s_{4}$ rejects his offer at $\sigma$ and participates in the second stage. Now, if student $s_{3}$ also rejects his offer and participates in the second stage, irrespective of the strategy that student $s_{1}$ chooses, student $s_{3}$ will receive $a$ and student $s_{4}$ will receive his outside option $s_{4}$. Since $a P_{s_{3}} \omega\left(s_{3}\right)$, student $s_{3}$ must also reject his offer in the equilibrium. But then, student $s_{4}$ accepting his offer and not participating in the second stage is a unilateral profitable deviation, contradicting that $\sigma$ is a Nash equilibrium. Hence, student $s_{4}$ accepts his offer at $\sigma$.

Now, if student $s_{3}$ also accepts his offer at $\sigma$, irrespective of the strategy that student $s_{1}$ chooses, student $s_{4}$ will receive $a$ if he rejects his offer and participates in the second stage, which would be a unilateral profitable deviation. Hence, student $s_{3}$ rejects his offer at $\sigma$.

Since $\omega\left(s_{3}\right) P_{s_{1}} \omega\left(s_{1}\right)$ and student $s_{1}$ has the top priority, student $s_{1}$ rejects his offer at $\sigma$ since he receives $\omega\left(s_{3}\right)$ by participating at the second stage.

Therefore, students $s_{1}, s_{2}$, and $s_{3}$ reject their offers and student $s_{4}$ accepts his offer at $\sigma$. But then, student $s_{3}$ receives his outside option $s_{3}$. Since $\omega\left(s_{3}\right) P_{s_{3}} s_{3}$, student $s_{3}$ has a profitable unilateral deviation, contradicting that $\sigma$ is a Nash equilibrium. Hence, the subgame does not have a Nash equilibrium.

Since there is a subgame without a pure-strategy Nash equilibrium, there is no purestrategy SPNE.

\section{Conclusion}

When designing a centralized clearinghouse to assign scarce resources based on priorities, such as in school choice, fairness is a critical desideratum. As a result, school districts that have reformed their admissions systems have employed the celebrated deferred acceptance (DA) algorithm. Even though the DA algorithm produces the student-optimal fair matching in one stage, in practice school districts have a second stage of assignment. Our study provides an explanation of why an additional stage of assignment may be 
preferable. In particular, an additional stage of assignment may improve student welfare if, and only if, the capacity-priority structure is not acyclic. Furthermore, in an environment where students are only strategic in acceptance-rejection decisions, having a second stage of assignment can never hurt any student.

Our analysis has the following policy implications. In an environment with an acyclic capacity-priority profile, the student enrollment systems should not have additional stages, as additional stages do not make students better off but may make them worse off. However, in an environment with a capacity-priority profile that has a cycle, the student welfare comparison can go both ways when students are sophisticated. However, if the capacity-priority profile can be designed, then it should be ayclic and the enrollment system should have only one stage. In this case, the 1-stage truthful outcome of DA Pareto dominates or is the same as any SPNE of the 2-stage enrollment system.

\section{References}

Abdulkadiroğlu, Atila and Tayfun Sönmez, "School choice: A mechanism design approach," American Economic Review, June 2003, 93 (3), 729-747.

Bando, Keisuke, "On the existence of a strictly strong Nash equilibrium under the studentoptimal deferred acceptance algorithm," Games and Economic Behavior, 2014, 87, 269-287.

Budish, Eric and Estelle Cantillon, "The Multi-unit Assignment Problem: Theory and Evidence from Course Allocation at Harvard," American Economic Review, May 2012, 102 (5), 2237-71.

Calsamiglia, Caterina, Guillaume Haeringer, and Flip Klijn, "Constrained School Choice: An Experimental Study," American Economic Review, September 2010, 100 (4), 1860-74.

Chambers, Christopher P. and M. Bumin Yenmez, "Choice and Matching," American Economic Journal: Microeconomics, 2017, 9, 126-147.

Chen, Yajing, "New axioms for deferred acceptance," Social Choice and Welfare, 2017, 48 (2), 393-408.

Combe, Julien, Olivier Tercieux, and Camille Terrier, "The Design of Teacher Assignment: Theory and Evidence," 2017. Working Paper.

Delacrétaz, David, Scott Duke Kominers, and Alexander Teytelboym, "Refugee Resettlement," 2016. Working Paper.

Doğan, Battal and Bettina Klaus, "Resource Allocation via Immediate Acceptance: Characterizations and an Affirmative Action Application," Journal of Mathematical Economics, forthcoming, 2018.

and M. Bumin Yenmez, "Unified versus Divided Enrollment in School Choice: Improving Student Welfare in Chicago," 2018. Working Paper. 
Dubins, Lester E. and David A. Freedman, "Machiavelli and the Gale-Shapley algorithm," The American Mathematical Monthly, 1981, 88 (7), 485-494.

Dur, Umut and Onur Kesten, "Sequential versus simultaneous assignment systems and two applications," Economic Theory, June 2018.

Echenique, Federico and M. Bumin Yenmez, "How to Control Controlled School Choice," American Economic Review, August 2015, 105 (8), 2679-2694.

Ergin, Haluk and Tayfun Sönmez, "Games of school choice under the Boston mechanism," Journal of Public Economics, 2006, 90, 215-237.

Ergin, Haluk I., "Consistency in house allocation problems," Journal of Mathematical Economics, 2000, 34 (1), 77 - 97.

, "Efficient resource allocation on the basis of priorities," Econometrica, 2002, 70 (6), 2489-2497.

Gale, David and Lloyd S. Shapley, "College Admissions and the Stability of Marriage," The American Mathematical Monthly, January 1962, 69 (1), 9-15.

Haeringer, Guillaume and Flip Klijn, "Constrained school choice," Journal of Economic Theory, 2009, 144 (5), 1921 - 1947. and Vincent Iehlé, "Gradual college admission," 2017. Working Paper.

Hylland, Aanund and Richard Zeckhauser, "The efficient allocation of individuals to positions," The Journal of Political Economy, 1979, 87 (2), 293-314.

Jackson, Matthew O., "Mechanism Theory," in Ulrich Derigs, ed., Optimization and Operations Research, Encyclopedia of Life Support Systems, Vol. 3, Oxford, U.K.: EOLSS, 2003.

Kelso, Alexander S. and Vincent P. Crawford, "Job Matching, Coalition Formation, and Gross Substitutes," Econometrica, 1982, 50, 1483-1504.

Kesten, Onur, "On two competing mechanisms for priority-based allocation problems," Journal of Economic Theory, 2006, 127 (1), 155 - 171.

, "School choice with consent," The Quarterly Journal of Economics, 2010, 125 (3), 1297-1348.

Kojima, Fuhito and M. Utku Ünver, "The "Boston" school-choice mechanism: an axiomatic approach," Economic Theory, April 2014, 55 (3), 515-544.

Manjunath, Vikram and Bertan Turhan, "Two school systems, one district: What to do when a unified admissions process is impossible," Games and Economic Behavior, 2016, 95, $25-40$.

Pycia, Marek, "Stability and Preference Alignment in Matching and Coalition Formation," Econometrica, 2012, 80 (1), 323-362.

Roth, Alvin E. and Marilda Sotomayor, Two-sided Matching: A Study in Game-Theoretic Modelling and Analysis, Vol. 18 of Econometric Society Monographs, Cambridge University Press, Cambridge England, 1990. 
Schummer, James and Rakesh V. Vohra, "Assignment of Arrival Slots," American Economic Journal: Microeconomics, 2013, 5 (2), 164-185.

Shapley, Lloyd and Herbert Scarf, "On cores and indivisibility," Journal of Mathematical Economics, 1974, 1 (1), 23 - 37.

Sönmez, Tayfun, "Bidding for Army Career Specialties: Improving the ROTC Branching Mechanism," Journal of Political Economy, 2013, 121 (1), 186-219.

and Tobias B. Switzer, "Matching With (Branch-of-Choice) Contracts at the United States Military Academy," Econometrica, 2013, 81 (2), 451-488.

and Utku Ünver, "Course Bidding at Business Schools," International Economic Review, 2010, 51 (1), 99-123. and , "House allocation with existing tenants: A characterization," Games and Economic Behavior, 2010, 69 (2), 425-445.

Sotomayor, Marilda, "The stability of the equilibrium outcomes in the admission games induced by stable matching rules," International Journal of Game Theory, 2008, 36, 621-640. Thomson, William, "The Consistency Principle," in Tatsuro Ichiishi, Abraham Neyman, and Yair Tauman, eds., Game Theory and Applications, New York: Academic Press, 1990, pp. 187-215.

Turhan, Bertan, "Welfare and incentives in partitioned school choice markets," Games and Economic Behavior, 2018.

\section{Appendix: Proofs}

In this section, we provide the omitted proofs. The next lemma is used in the proof of Theorem 1, which is shown in Chen (2017) and called "weak consistency." We provide a proof for completeness.

Lemma 1. Suppose that DA produces matching $\mu$. When a set of students is removed and the capacities of their assigned schools in $\mu$ are reduced accordingly, the remaining students receive weakly more preferred outcomes at DA of the new problem.

The mathematical formulation of the result is as follows: For each problem $\left(\mathcal{S}, \mathcal{C},\left(R_{s}\right)_{s \in \mathcal{S}},\left(q_{c}, \succeq_{c}\right)_{c \in \mathcal{C}}\right), S \subseteq \mathcal{S}$, and $s \in \mathcal{S} \backslash S$, the outcome of DA under $(\mathcal{S} \backslash$ $\left.S, \mathcal{C},\left(R_{S}\right)_{s \in \mathcal{S} \backslash S},\left(q_{c}-|\mu(c) \cap S|, \succeq_{c}\right)_{c \in \mathcal{C}}\right)$ is weakly more preferred with respect to $R_{S}$ than the outcome of DA under $\left(\mathcal{S}, \mathcal{C},\left(R_{S}\right)_{s \in \mathcal{S}},\left(q_{c}, \succeq_{c}\right)_{c \in \mathcal{C}}\right)$.

Proof of Lemma 1. Let $\mu^{\prime}$ be the matching obtained from $\mu$ by removing the set of students $S$ together with their assigned seats at $\mu$. Note that matching $\mu^{\prime}$ includes only the students in $\mathcal{S} \backslash S$. First we show that $\mu^{\prime}$ is a stable matching in the new problem $\left(\mathcal{S} \backslash S, \mathcal{C},\left(R_{s}\right)_{s \in \mathcal{S} \backslash S},\left(q_{c}-|\mu(c) \cap S|, \succ_{c}\right)_{c \in \mathcal{C}}\right)$. Individual rationality for students holds since $\mu$ is individually rational in $\left(\mathcal{S}, \mathcal{C},\left(R_{s}\right)_{s \in \mathcal{S}},\left(q_{c}, \succ_{c}\right)_{c \in \mathcal{C}}\right)$. For any school $c$, individual 
rationality in $\left(\mathcal{S}, \mathcal{C},\left(R_{S}\right)_{s \in \mathcal{S}},\left(q_{c}, \succ_{c}\right)_{c \in \mathcal{C}}\right)$ implies that $C h_{c}\left(\mu(c) ; q_{c}\right)=\mu(c)$. In $\mu^{\prime}$, the set of students matched to school $c$ is $\mu(c) \backslash S$ and the capacity of $c$ is $q_{c}-|\mu(c) \cap S|$. Since $C h_{c}$ is the responsive choice function, we get $C h_{c}\left(\mu(c) \backslash S ; q_{c}-|\mu(c) \cap S|\right)=\mu(c) \backslash S$, which shows that $\mu^{\prime}$ is individually rational for school $c$. Any blocking pair $(s, c)$ for $\mu^{\prime}$ in the new problem $\left(\mathcal{S} \backslash S, \mathcal{C},\left(R_{s}\right)_{s \in \mathcal{S} \backslash S^{\prime}}\left(q_{c}-|\mu(c) \cap S|, \succ_{c}\right)_{c \in \mathcal{C}}\right)$ would also be a blocking pair in the original problem $\left(\mathcal{S}, \mathcal{C},\left(R_{S}\right)_{s \in \mathcal{S}},\left(q_{c}, \succ_{c}\right)_{c \in \mathcal{C}}\right)$ because of responsiveness, so blocking pairs cannot exist. Therefore, $\mu^{\prime}$ is stable in the new problem.

Since $\mu^{\prime}$ is stable in the new problem, the outcome of DA for the new problem is weakly more preferred for the remaining students since DA produces the student-optimal stable matching (Gale and Shapley, 1962). The conclusion follows.

Proof of Theorem 1. Lemma 1 shows that removing students and their allotments in DA weakly improves the outcome for the remaining students in DA. Since students submit their preferences truthfully in both stages of the 2-stage enrollment system, and since some students may leave by accepting their first-stage offers, the outcome of DA improves weakly at the second stage for all the remaining students. Therefore, a student gets a weakly more preferred school by rejecting the first-stage offer. Hence, at any strategy profile of the 2-stage system, a student $s$ either accepts his offer at the first stage and is assigned to his 1-stage enrollment school, or some students leave with their 1-stage enrollment schools and in the second stage student $s$ is assigned a weakly better school by Lemma 1.

Proof of Theorem 2. Part $i$. Since the capacity-priority profile is acylic, DA is consistent. Therefore, for any student, accepting and rejecting the first-stage offer are outcome equivalent. Hence, any strategy profile is an equilibrium, which produces the same outcome as DA.

Part ii. Let $\left(q_{c}, \succeq_{c}\right)_{c \in \mathcal{C}}$ be a capacity-priority profile that has a cycle. Then, there exist a pair of schools $c$ and $c^{\prime}$, and three students $s, s^{\prime}$, and $s^{\prime \prime}$ such that

i. $s \succ_{c} s^{\prime} \succ_{c} s^{\prime \prime} \succ_{c^{\prime}} s$ and

ii. there exist (possibly empty) disjoint sets of students $S_{c}, S_{c^{\prime}} \subset \mathcal{S} \backslash\left\{s, s^{\prime}, s^{\prime \prime}\right\}$ with $S_{c} \subset\left\{i \in S: i \succ_{c} s^{\prime}\right\}, S_{c^{\prime}} \subset\left\{i \in S: i \succ_{c^{\prime}} s\right\},\left|S_{c}\right|=q_{c}-1$, and $\left|S_{c^{\prime}}\right|=q_{c^{\prime}}-1$.

Consider a preference profile $\left(R_{S}\right)_{s \in \mathcal{S}}$ such that

i. $c^{\prime} P_{S} c P_{S} S P_{S} c^{\prime \prime}$ for every $c^{\prime \prime} \in \mathcal{C} \backslash\left\{c, c^{\prime}\right\}$,

ii. $c P_{s^{\prime}} s^{\prime} P_{s^{\prime}} c^{\prime \prime}$ for every $c^{\prime \prime} \in \mathcal{C} \backslash\{c\}$,

iii. $c P_{s^{\prime \prime}} c^{\prime} P_{s^{\prime \prime}} s^{\prime \prime} P_{s^{\prime \prime}} c^{\prime \prime}$ for every $c^{\prime \prime} \in \mathcal{C} \backslash\left\{c, c^{\prime}\right\}$,

iv. $c P_{i} i P_{i} c^{\prime \prime}$ for every $i \in S_{c}$ and every $c^{\prime \prime} \in \mathcal{C} \backslash\{c\}$,

v. $c^{\prime} P_{i} i P_{i} c^{\prime \prime}$ for every $i \in S_{c^{\prime}}$ and every $c^{\prime \prime} \in \mathcal{C} \backslash\left\{c^{\prime}\right\}$, and

vi. $i P_{i} c^{\prime \prime}$ for every $i \in \mathcal{S} \backslash\left\{\left\{s, s^{\prime}, s^{\prime \prime}\right\} \cup S_{c} \cup S_{c^{\prime}}\right\}$ and every $c^{\prime \prime} \in \mathcal{C}$. 
The relevant part of the restriction of the preference relation profile and the priority profile to $\left\{s, s^{\prime}, s^{\prime \prime}, c, c^{\prime}\right\}$ looks as follows.

\begin{tabular}{cccccc}
$R_{S}$ & $R_{s^{\prime}}$ & $R_{s^{\prime \prime}}$ & & $\succeq_{c}$ & $\succeq_{c^{\prime}}$ \\
\cline { 1 - 3 }$c^{\prime}$ & $c$ & $c$ & & $s$ & $s^{\prime \prime}$ \\
$c$ & $s^{\prime}$ & $c^{\prime}$ & & $s^{\prime}$ & $s$ \\
$s$ & & $s^{\prime \prime}$ & & $s^{\prime \prime}$ &
\end{tabular}

We construct a Nash equilibrium of the 2-stage enrollment system for the problem $\left(R_{S}\right)_{s \in \mathcal{S}}$ that Pareto dominates the truthful equilibrium of the 1-stage enrollment system. In the truthful outcome of the 1-stage enrollment system, students in $\{s\} \cup S_{c}$ get matched with $c$, and students in $\left\{s^{\prime}\right\} \cup S_{c^{\prime}}$ get matched with $c^{\prime}$, whereas the remaining students (including $s^{\prime}$ ) get their outside options.

Consider the following strategy profile $\sigma$ for the 2-stage enrollment system in which students $s$ and $s^{\prime \prime}$ reject and every other student accepts their offers. Therefore, only students $s$ and $s^{\prime \prime}$ participate in the second stage with schools $c$ and $c^{\prime}$, both of which have a remaining capacity of only one. At the second stage, $s$ applies to $c^{\prime}$ and $s^{\prime \prime}$ applies to $c$, both of which are accepted. In the outcome under the strategy profile $\sigma$, students in $\left\{s, s^{\prime \prime}\right\} \cup S_{c} \cup S_{c^{\prime}}$ get their most preferred schools, whereas the other students get their outisde options. Therefore, the outcome of the 2-stage enrollment system under $\sigma$ Pareto dominates the truthful outcome of the 1-stage enrollment system.

We now show that $\sigma$ is a Nash equilibrium of the 2-stage enrollment system. Every student other than $s^{\prime}$ gets their most preferred outcome. Furthermore, if student $s^{\prime}$ deviates and rejects her offer, then students $s, s^{\prime}$, and $s^{\prime \prime}$ participate in the second stage with schools $c$ and $c^{\prime}$ with remaining capacity of one. Then the outcome of DA at the second stage matches $s^{\prime}$ with her outside option, so student $s^{\prime}$ does not have a unilateral deviation in which she is matched with a more preferred outcome. Therefore, $\sigma$ is a Nash equilibrium of the 2-stage enrollment system.

Proof of Theorem 3. Consider any SPNE outcome of the 2-stage enrollment system, say $\mu$. We first show that there is no blocking pair. Suppose, for contradiction, that there exist students $s, s^{\prime}$ and school $c$ such that $\mu\left(s^{\prime}\right)=c, c P_{s} \mu(s)$, and $s \succ_{c} s^{\prime}$. Suppose, without loss of generality, that student $s^{\prime}$ is the lowest-priority student at school $c$ in matching $\mu$.

Case 1: Both students $s$ and $s^{\prime}$ accept their offers and get matched at the first stage. Let $\tilde{R}_{s}$ denote the preference relation that student $s$ reports. Since DA is used, the school assigned to student $s$ is better than school $c$ with respect to preference relation $\tilde{R}_{s}$.

Consider a one-shot deviation of student $s$ at the preference-revelation decision node where she instead reports another preference relation, say $\tilde{R}_{s}^{\prime}$, which is obtained from $\tilde{R}_{s}$ just by moving school $c$ to the top. 
Consider the subcase when student $s$ receives school $c$ at the first stage. In the following subgame, his strategy either recommends he accept school $c$ or recommends he reject school $c$, in which case he receives a school at least as good as school $c$ at the end of the game, since his strategy induces a Nash equilibrium in every subgame. In any case, there is a profitable one-shot deviation, which is a contradiction.

Consider the other subcase when student $s$ does not receive school $c$ at the first stage. Since there cannot be any interrupter, in any step of DA where student $s$ is tentatively accepted by school $c$, no other student is rejected by $c$ at the same step. And, as a result, DA produces the same matching as when he reports $\tilde{R}_{s}$. Therefore, student $s^{\prime}$ is matched with school $c$, which contradicts the stability of DA.

Case 2: Student s' accepts his offer and student s rejects his offer at the first stage. Then, the school that made student $s$ an offer in the first stage, if any, must be worse than school $c$, since otherwise student $s$ would have a profitable one-shot deviation at an accept-reject decision node where he instead accepts his offer. But then, due to the same arguments as in Case 1, student $s$ has a profitable one-shot deviation at the preference-revelation decision node by moving school $c$ to the top of his preference ranking.

Case 3: Student s accepts his offer and student s' rejects his offer at the first stage. Consider a one-shot deviation of student $s$ at the accept-reject decision node where he instead rejects his offer.

Now, in the second stage, there will be one additional student, $s$, and at most one more seat for a school. Consider the subproblem without the additional seat, but only with the additional student $s$. First note that there cannot be an interrupter in this problem. This is not immediately implied by the acyclic capacity-priority profile assumption, since we are looking at a subproblem where the capacities of the schools may be smaller than their original capacities. However, by the argument in Case 2, for each student who receives a seat in Stage 2, and for each school that he prefers to his assigned school in Stage 2, all the students who receive that school in the first stage must have a higher priority. Now, consider the problem obtained from the subproblem by adding the students who received seats in the first stage and the seats that were allocated in the first stage, with the new preference profile where the additional students find acceptable only the school that they were assigned in the first stage. Clearly, any student who is an interrupter in the subproblem will still be an interrupter in this new problem when the schools have their original capacities, which is not possible since this new problem has the same capacitypriority profile with the original problem, which is an acyclic capacity-priority profile. Hence, there is no interrupter in the subproblem.

Next, we will show that, since initially student $s^{\prime}$ receives school $c$ and student $s$ has higher priority than student $s^{\prime}$ at school $c$, student $s$ gets either school $c$ or something 
better in the subproblem after his one-shot deviation. Suppose, for contradiction, that student $s$ gets a school that is worse than school $c$. For any step of DA at which student $s$ is tentatively accepted by a school that he prefers to school $c$, no other student should be rejected from that school at the same step, since there is no interrupter. Also, for any step of DA at which student $s$ is tentatively accepted by school $c$, no other student should be rejected from $c$ at the same step. Now, consider the step at which $s$ is rejected by $c$. Note that until student $s$ gets rejected by school $c$, DA if student $s$ had not deviated to participate in the second stage and DA if he deviated to participate in the second stage run exactly the same. Also note that, since student $s$ gets a school that is worse than school $c$ and the allocation must be stable, student $s^{\prime}$ must be rejected from school $c$ at some step. If student $s^{\prime}$ is tentatively accepted at school $c$ in the same step at which student $s$ gets rejected by school $c$, then student $s$ is an interrupter at school $c$, which is a contradiction. Suppose that student $s^{\prime}$ is not one of the tentatively accepted students at school $c$ in the step that student $s$ gets rejected by school $c$. Then, there exists a student $s^{\prime \prime}$ who has lower priority than student $s^{\prime}$ at school $c$ and gets tentatively accepted by school $c$ at the step student $s$ gets rejected by school $c$, since in DA when student $s$ had not deviated to participate in the second stage, student $s^{\prime}$ applies to school $c$ at a later step and gets tentatively accepted. But then, student $s^{\prime \prime}$ gets rejected by school $c$ at a later step, by the latest at the first step student $s^{\prime}$ applies to school $c$. Hence, student $s^{\prime \prime}$ is an interrupter at school $c$, which is a contradiction.

Thus, student $s$ gets either school $c$ or something better after his one-shot deviation in the subproblem without the added seat. By resource monotonicity of DA (Kelso and Crawford, 1982; Chambers and Yenmez, 2017), we conclude that student $s$ gets either school $c$ or something better after his one-shot deviation, which is a profitable one-shot deviation. Thus, we get a contradiction.

Case 4: Students s and s' accept their offers at the second stage. Since students submit their true preferences at the second stage and DA is stable, student $s^{\prime}$ cannot be matched with school $c$ while student $s$ does not receive an offer from school $c$ or a better school with respect to his preference ranking $R_{s}$ because student $s$ has a higher priority than student $s^{\prime}$ at school $c$. This is a contradiction.

In all possible cases, we have established a contradiction. Hence, matching $\mu$ has no blocking pairs. In addition, it must be individually rational since in equilibrium a student does not accept an unacceptable school and a school never chooses unacceptable students. Yet this does not mean that matching $\mu$ is stable because it can have waste: a student may prefer an empty seat to his assigned seat. Consider the reduced problem obtained by decreasing the capacities of the schools (by the amount of empty seats). Then, matching $\mu$ is stable at this new problem. Let matching $\mu^{*}$ be the student-optimal stable matching 
at this problem. Note that each student is weakly better off at matching $\mu^{*}$ compared to matching $\mu$. Also, by resource monotonicity of the DA, when the school capacities are increased to the initial levels, which is the 1-stage enrollment outcome, each student must be weakly better off, compared to $\mu^{*}$ and also compared to $\mu$. Hence, there is no student at $\mu$ who is better off compared to the outcome of the 1-stage enrollment system.

Proof of Theorem 4. Let $\left(q_{c}, \succeq_{c}\right)_{c \in \mathcal{C}}$ be a capacity-priority profile that is not acyclic. Then, there exist a pair of schools $c$ and $c^{\prime}$, and three students $s, s^{\prime}$, and $s^{\prime \prime}$ such that

i. $s \succ_{c} s^{\prime} \succ_{c} s^{\prime \prime} \succ_{c^{\prime}} s$ and

ii. there exist (possibly empty) disjoint sets of students $S_{c}, S_{c^{\prime}} \subset \mathcal{S} \backslash\left\{s, s^{\prime}, s^{\prime \prime}\right\}$ such that $S_{c} \subset\left\{i \in S: i \succ_{c} s^{\prime}\right\}, S_{c^{\prime}} \subset\left\{i \in S: i \succ_{c^{\prime}} s\right\},\left|S_{c}\right|=q_{c}-1$, and $\left|S_{c^{\prime}}\right|=q_{c^{\prime}}-1$.

Consider a preference profile $\left(R_{s}\right)_{s \in \mathcal{S}}$ such that

i. $c^{\prime} P_{S} c P_{S} s P_{S} c^{\prime \prime}$ for every $c^{\prime \prime} \in \mathcal{C} \backslash\left\{c, c^{\prime}\right\}$,

ii. $c P_{s^{\prime}} s^{\prime} P_{s^{\prime}} c^{\prime \prime}$ for every $c^{\prime \prime} \in \mathcal{C} \backslash\{c\}$,

iii. $c P_{s^{\prime \prime}} c^{\prime} P_{s^{\prime \prime}} s^{\prime \prime} P_{s^{\prime \prime}} c^{\prime \prime}$ for every $c^{\prime \prime} \in \mathcal{C} \backslash\left\{c, c^{\prime}\right\}$,

iv. $c P_{i}$ i $P_{i} c^{\prime \prime}$ for every $i \in S_{c}$ and every $c^{\prime \prime} \in \mathcal{C} \backslash\{c\}$,

v. $c^{\prime} P_{i} i P_{i} c^{\prime \prime}$ for every $i \in S_{c^{\prime}}$ and every $c^{\prime \prime} \in \mathcal{C} \backslash\left\{c^{\prime}\right\}$, and

vi. $i P_{i} c^{\prime \prime}$ for every $i \in \mathcal{S} \backslash\left\{\left\{s, s^{\prime}, s^{\prime \prime}\right\} \cup S_{c} \cup S_{c^{\prime}}\right\}$ and every $c^{\prime \prime} \in \mathcal{C}$.

The relevant part of the restriction of the preference relation profile and the priority profile to $\left\{s, s^{\prime}, s^{\prime \prime}, c, c^{\prime}\right\}$ looks as follows.

\begin{tabular}{cccccc}
$R_{s}$ & $R_{s^{\prime}}$ & $R_{s^{\prime \prime}}$ & & $\succeq_{c}$ & $\succeq_{c^{\prime}}$ \\
\cline { 1 - 3 }$c^{\prime}$ & $c$ & $c$ & & $s$ & $s^{\prime \prime}$ \\
$c$ & $s^{\prime}$ & $c^{\prime}$ & & $s^{\prime}$ & $s$ \\
$s$ & & $s^{\prime \prime}$ & & $s^{\prime \prime}$ &
\end{tabular}

We will construct an SPNE of the 2-stage enrollment system for the problem $\left(R_{s}\right)_{s \in \mathcal{S}}$ that Pareto dominates the truthful equilibrium of the 1-stage enrollment system. The construction generalizes the idea in Example 2, and, essentially, students $s, s^{\prime}$, and $s^{\prime \prime}$ play the roles of students $s_{3}, s_{1}$, and $s_{2}$ in Example 2, respectively, schools $c$ and $c^{\prime}$ play the roles of schools $c_{2}$ and $c_{1}$ in Example 2, respectively, and the outside option of student $s_{2}$ plays the role of school $c_{3}$ in Example 2. As will be seen shortly, the strategy profile in Example 2 will need to be modified due to the additional students $S_{c}$ and $S_{c^{\prime}}$.

First note that in the 1-stage enrollment outcome, students in $\{s\} \cup S_{c}$ get school $c$, students in $\left\{s^{\prime \prime}\right\} \cup S_{c^{\prime}}$ get school $c^{\prime}$, and every other student gets his outside option. Consider the following strategy profile for the 2-stage enrollment system:

i. Student $s^{\prime}$ reports no school acceptable in the first stage and accepts his offer if, and only if, it is strictly preferred to his 1-stage enrollment school, which is his outside option $s^{\prime}$. 
ii. Student $s^{\prime \prime}$ reports truthfully in the first stage and accepts his offer if, and only if, - either it is strictly preferred to his 1-stage enrollment school,

- or it is his 1-stage enrollment school and "student $s$ has an offer from $c^{\prime}$ or student $s^{\prime}$ has an offer from $c . "$

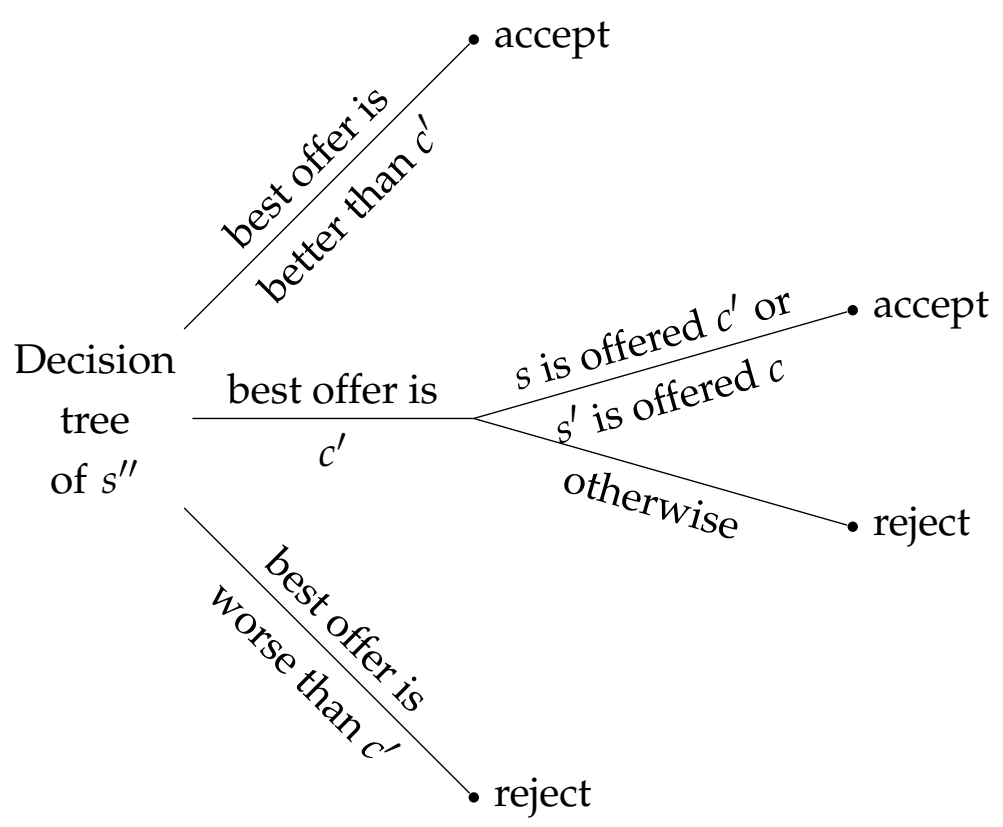

iii. Student $s$ reports truthfully in the first stage and accepts his offer if, and only if,

- either it is strictly preferred to his 1-stage enrollment school,

- or it is his 1-stage enrollment school and "student $s^{\prime}$ has an offer from $c$ and student $s^{\prime \prime}$ does not have an offer from $c . "$

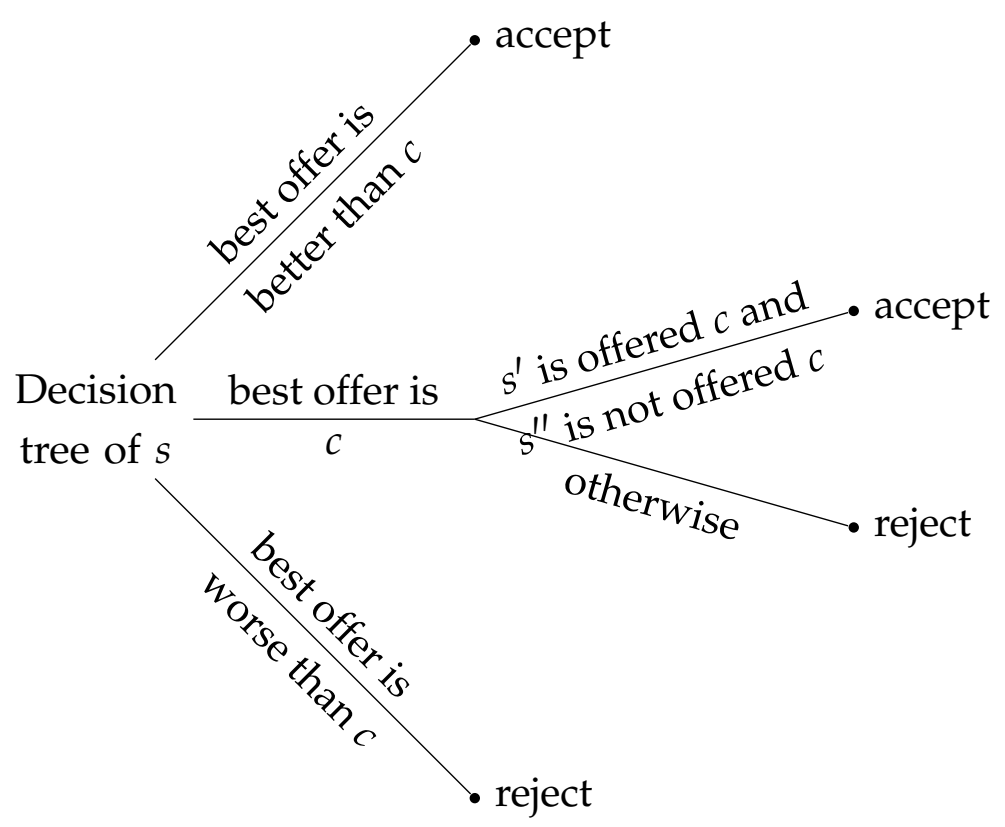


iv. Each other student in $\mathcal{S} \backslash\left\{s, s^{\prime}, s^{\prime \prime}\right\}$ reports truthfully in the first stage and accepts his offer if, and only if, it is weakly preferred to his 1-stage enrollment school.

Consider student $s^{\prime}$. By following the above strategy, he gets his outside option. His only acceptable school is $c$. By unilaterally changing his preference report, even if he applies to school $c$, he will not get an offer from $c$ and he will make students $s$ and $s^{\prime \prime}$ get their best offers from their 1-stage enrollment schools, in which case both $s$ and $s^{\prime \prime}$ reject their offers. Then, in the second stage, student $s^{\prime}$ will get his outside option, while $s$ and those students in $S_{c}$ who did not get an offer from $c$ and accepted in the first stage will occupy the remaining seats of $c$. So there is no one-shot profitable deviation at his preference-revelation decision node. Consider any of his acceptance-rejection decision nodes. If he is offered school $c$, clearly he can not be strictly better off by rejecting it. If he is offered his outside option or any school worse than that, and he rejects, in the worst case he receives his outside option in the second stage, so again there is no one-shot profitable deviation.

Consider student $s^{\prime \prime}$. By following the above strategy, he gets school $c$, which is his top choice. In the preference-revelation decision node, there is no one-shot profitable deviation since he gets his best choice by following the prescribed strategy. Consider any of his acceptance-rejection decision nodes. If he is offered a school strictly better than $c^{\prime}$ or strictly worse than $c^{\prime}$, clearly he cannot be better off by a one-shot deviation at that decision node. Suppose that he is offered school $c^{\prime}$.

Case 1: $s$ is offered $c^{\prime}$ and $s^{\prime}$ is offered $c$. The prescribed strategy recommends $s^{\prime \prime}$ accept $c^{\prime}$. If he instead rejects, he cannot get $c$ in the second stage since $s^{\prime}$ accepts $c$ in the first stage, and, in the second stage, the remaining seats of $c$ are taken by those students in $S_{c}$ who did not get an offer from $c$ in the first stage. Therefore there is no one-shot profitable deviation.

Case 2: $s$ is offered $c^{\prime}$ and $s^{\prime}$ is not offered $c$. The prescribed strategy recommends $s^{\prime \prime}$ to accept $c^{\prime}$. If he instead rejects, he cannot get $c$ in the second stage since $s$ accepts $c^{\prime}$ and $s^{\prime}$ rejects his best offer in the first stage, and in the second stage seats of $c$ are taken by $s^{\prime}$ and those students in $S_{c}$ who did not get an offer from $c$ in the first stage. Therefore there is no one-shot profitable deviation.

Case 3: $s$ is not offered $c^{\prime}$ and $s^{\prime}$ is offered $c$. The prescribed strategy recommends $s^{\prime \prime}$ to accept $c^{\prime}$. If $s$ is offered $c$, both $s$ and $s^{\prime}$ accept their offers, and $s^{\prime \prime}$ cannot get $c$ in the second stage since the remaining seats of $c$ are taken by those students in $S_{c}$ who did not receive an offer from $c$ in the first stage. If $s$ is offered a school different than $c$ and $c^{\prime}$, then $s^{\prime}$ accepts $c, s$ rejects his best offer, and $s^{\prime \prime}$ cannot get $c$ in the second stage since the remaining seats of $c$ are taken by those students in $S_{c} \cup\{s\}$ who did not receive an offer from $c$ in the first stage, except for the lowest-priority one (who may or may not be student s). Therefore, there is no one-shot profitable deviation. 
Case 4: $s$ is not offered $c^{\prime}$ and $s^{\prime}$ is not offered $c$. The prescribed strategy recommends $s^{\prime \prime}$ reject $c^{\prime}$, in which case $s^{\prime \prime}$ eventually gets $c^{\prime}$, which is equivalent to accepting. Therefore there is no one-shot profitable deviation.

Consider student $s$. By following the above strategy, he gets school $c^{\prime}$, which is his top choice. In the preference-revelation decision node, there is no one-shot profitable deviation since he gets his best choice by following the prescribed strategy. Consider any of his acceptance-rejection decision nodes. If he is offered a school strictly better than $c$ or strictly worse than $c$, clearly he cannot be better off by a one-shot deviation at that decision node. Suppose that he is offered school $c$.

Case 1: $s^{\prime}$ is offered $c$ and $s^{\prime \prime}$ is not offered $c$. The prescribed strategy recommends $s$ accept $c$. Suppose that $s^{\prime \prime}$ is offered offered $c^{\prime}$. If $s^{\prime}$ instead rejects, he cannot get $c^{\prime}$ in the second stage since $s^{\prime \prime}$ accepts $c^{\prime}$ in the first stage and in the second stage, the remaining seats of $c^{\prime}$ are taken by those students in $S_{c^{\prime}}$ who did not get an offer from $c^{\prime}$ in the first stage. Suppose that $s^{\prime \prime}$ is offered a school worse than $c^{\prime}$. If $s^{\prime}$ instead rejects, he still cannot get $c^{\prime}$ in the second stage since $s^{\prime \prime}$ rejects his offer and $s^{\prime}$ accepts $c$ in the first stage, and, in the second stage in the DA algorithm, $s^{\prime \prime}$ will be rejected by $c$ in the first step, and in turn student $s$ will be rejected by $c^{\prime}$ in the second step. Therefore, there is no one-shot profitable deviation.

Case 2: $s^{\prime}$ is offered $c$ and $s^{\prime \prime}$ is also offered $c$. The prescribed strategy recommends $s$ reject $c$. Since both $s^{\prime}$ and $s^{\prime \prime}$ accept their offers from $c$, in the second stage student $s$ gets $c^{\prime}$ after rejecting in the first stage. Therefore, there is no one-shot profitable deviation.

Case 3: $s^{\prime}$ is not offered $c$. The prescribed strategy recommends $s$ reject $c$. If $s^{\prime \prime}$ is offered $c$, then $s^{\prime \prime}$ accepts the offer, and, in the second stage, $s$ gets $c^{\prime}$, which he prefers to $c$, together with those students in $S_{c^{\prime}}$ who did not get an offer from $c^{\prime}$ in the first stage. If $s^{\prime \prime}$ is offered $c^{\prime}, s^{\prime \prime}$ accepts $c^{\prime}$ and in the second stage $s^{\prime}$ gets $c$. If $s^{\prime \prime}$ is offered a school different than $c$ and $c^{\prime}$, then both $s^{\prime}$ and $s^{\prime \prime}$ reject their offers, and, in the second stage, $s$ gets $c$ together with those students in $S_{c}$ who did not get an offer from $c$ in the first stage. Therefore, there is no one-shot profitable deviation.

Consider any student $s^{*} \in \mathcal{S} \backslash\left\{s, s^{\prime}, s^{\prime \prime}\right\}$. By following the above strategy, he gets his top choice. In the preference-revelation decision node, there is no one-shot profitable deviation since he gets his best choice by following the prescribed strategy. Consider any of his acceptance-rejection decision nodes. If he is offered his top choice, the prescribed strategy recommends $s^{*}$ accept it, in which case, clearly there is no one-shot profitable deviation. If he is not offered his top choice, then it must be that $s^{*} \in S_{c} \cup S_{c^{\prime}}$, and by rejecting $s^{*}$ can ensure an outcome at least as good as his outside option, which is his second-ranked school and therefore at least as good as his first-stage offer. Therefore, there is no one-shot profitable deviation. 